\title{
Pulling-Force Spinning Top for Serum Separation Combined with Paper-Based Microfluidic Devices in COVID-19 ELISA Diagnosis
}

Fanwu Gong, Hua-xing Wei, Ji Qi, Huan Ma, Lianxin Liu, Jianping Weng, Xucai Zheng, Qiangsheng Li, Dan Zhao, Haopeng Fang, Liu Liu, Hongliang He, Cuichen Ma, Jinglong Han, Anyuan Sun, Baolong Wang, Tengchuan Jin,* Bowei $\mathrm{Li}^{*}$ * and Bofeng $\mathrm{Li}^{*}$

Cite This: ACS Sens. 2021, 6, 2709-2719

Read Online

Supporting Information

ABSTRACT: The spread of Coronavirus disease 2019 (COVID-19) is caused by severe acute respiratory syndrome-coronavirus 2 (SARS-CoV-2), resulting in a global pandemic with around four million deaths. Although there are a variety of nucleic acid-based tests for detecting SARS-CoV-2, these methods have a relatively high cost and require expensive supporting equipment. To overcome these limitations and improve the efficiency of SARS-CoV-2 diagnosis, we developed a microfluidic platform that collected serum by a pulling-force spinning top and paper-based microfluidic enzyme-linked immunosorbent assay (ELISA) for quantitative $\operatorname{IgA} / \operatorname{IgM} / \operatorname{IgG}$ measurements in an instrument-free way. We further validated the paper-based microfluidic ELISA analysis of SARS-CoV-2 receptorbinding domain (RBD)-specific IgA/IgM/IgG antibodies from human blood samples as a good measurement with higher sensitivity compared with traditional IgM/IgG detection ( $99.7 \%$ vs $95.6 \%$ ) for early illness onset patients. In conclusion,

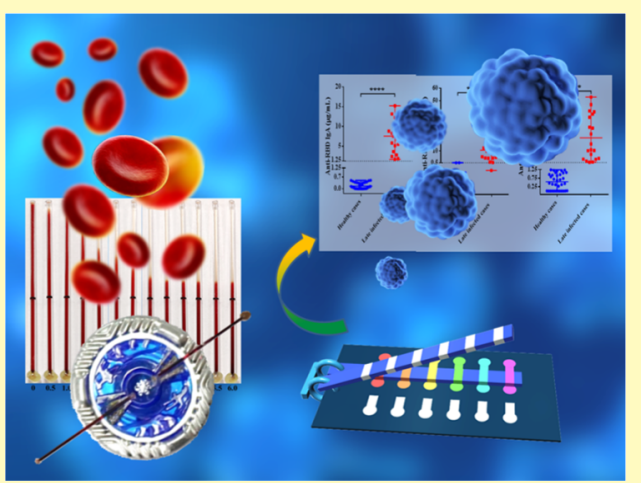
we provide an alternative solution for the diagnosis of SARS-CoV-2 in a portable manner by this smart integration of pulling-force spinning top and paper-based microfluidic immunoassay.

KEYWORDS: SARS-Cov-2, COVID-19 diagnosis, paper-based ELISA, anti-RBD antibody, serum separation, microfluidic devices

Coror oronavirus infection leads to a public health crisis since the beginning of the 21 st century, ${ }^{1}$ including the appearance of severe acute respiratory syndrome-coronavirus (SARS-CoV) in 2003 and Middle East respiratory syndrome-coronavirus (MERS-CoV) in 2012, especially the new emergency of coronavirus disease 2019 (COVID-19), which has spread rapidly all over the world. ${ }^{2,3}$ After infected with SARS-CoV-2, patients have respiratory symptoms, such as cough, fever, and shortness of breath. Moreover, patients with severe disease could develop pneumonia, kidney failure, and brain failure or even die. ${ }^{4}$ The current global coronavirus epidemic is progressing quickly, causing a huge health and economic problem. To date, the total number of infected people has exceeded 173000000 , as well as more than 3700000 deaths.

Therefore, there is an urgent need for a more reliable and cheap diagnostic method to screen and distinguish the SARSCoV-2-infected patients from healthy people. To date, most clinical inspections of the SARS-CoV-2 virus are based on the real-time polymerase chain reaction (PCR) analysis of nasopharyngeal or throat swab samples or enzyme-linked immunosorbent assay (ELISA) about detecting the expression of virus protein. There is always a concern about the difficulty of obtaining high-quality throat swab samples, and comparative lower viral load at the early stage of infection, resulting in a high false-negative rate. ${ }^{5,6}$ On the other hand, lateral flow rapid test strip gives a way to confirm the presence or absence of the SARS$\mathrm{CoV}-2$ virus, but it cannot provide quantitative information and lead to a limitation in clinical practice.

Many people in developed countries have been infected with SARS-CoV-2, such as the United States, United Kingdom, Italy, etc. In addition, more than 100 other countries and regions around the world have also found COVID-19 patients, including many third-world countries. ${ }^{7,8}$ These third-world countries have large populations, high population density, and insufficient medical resources. Although there are a variety of reverse transcription polymerase chain reaction (RT-PCR) kits for detecting SARS-CoV-2, the RT-PCR method has a relatively high cost and requires expensive supporting equipment. It is crucial to develop some new methods to achieve low-cost and fast detection of SARS-CoV-2. ${ }^{5}$

Recently, microfluidic paper-based analytical devices ( $\mu$ PADs) have been a hotspot of research due to their multiple

Received: April 14, 2021

Accepted: June 28, 2021

Published: July 15, 2021 
inherent advantages, such as low cost, small reagent consumption, no external power source, and good biocompatibility. ${ }^{9-11}$ Therefore, microfluidic paper chips are increasingly being used in many fields including point-of-care testing (POCT), ${ }^{9,12}$ environmental monitor, ${ }^{13,14}$ clinical analysis, ${ }^{9,15}$ food processing, and chemical industries. ${ }^{16}$ For example, Yu's group used three-dimensional paper-based electrochemiluminescence and chemiluminescence immunoassays to develop a series of methods for the measurement and rapid testing of biomarkers. ${ }^{17-19}$ Bhamla and his co-workers invented a paper centrifugation technology that worked without electricity and separated blood in a few minutes. ${ }^{20}$ Using a high-speed centrifugal rotation of the fidget spinner, Michael made a rapid diagnosis of urinary tract infection ${ }^{21}$ and Liu realized the human immunodeficiency virus (HIV-1) detection. ${ }^{22}$ We also provided integrated hand-powered centrifugation-combined ELISA diagnosis on a microfluidic device. ${ }^{9}$

As is well known, mature SARS-CoV-2 contains four conserved structural proteins: spike $(S)$, envelope $(E)$, membrane $(\mathrm{M})$, and nucleocapsid protein $(\mathrm{N})$, as well as several accessory proteins. ${ }^{7,23}$ More importantly, $\mathrm{S}$ protein contains S1 and S2 subunits, while receptor-binding domain (RBD) as part of S1 submit can bind to human angiotensinconverting enzyme 2 (ACE2) receptor, thus playing an essential role for the entrance into the cell membrane. ${ }^{24,25}$ When the virus invades the host, the immune system produces large amounts of immunoglobulins ( $\operatorname{IgG}, \operatorname{IgM}$, and $\operatorname{IgA}$ ), which were released into the blood. ${ }^{26,27}$ Now, detecting RBD-specific antibody productions is a novel serological method and has unique advantages in clinical diagnostics, especially for identifying people who have acquired immunity against pathogens without noticeable symptoms. ${ }^{5}$ It has been widely believed that IgM is the first antibody to be transiently synthesized in response to the virus invasion. ${ }^{28}$ IgG is a major class of immunoglobulins found in the blood, which is induced by a secondary immune response and has long-term immunity and immunological memory. ${ }^{29,30}$ Therefore, a traditional IgM/IgG combination was used to diagnose the SARS-CoV-2 infection initially; however, the effect was questionable. Our previous study found that IgA also appeared at the early stage of the disease, ${ }^{31}$ starting to be used as a good marker in the detection of SARS-CoV-2. ${ }^{3,33}$

We had successfully built a serological solution to monitor the infection of SARS-CoV-2 virus by detecting the serum RBDspecific $\operatorname{IgA} / \operatorname{IgM} / \operatorname{IgG}$ productions and proved that the combination of these parameters greatly increased the sensitivity of the diagnosis. ${ }^{31}$ However, this method still requires the apparatus (automatic chemical luminescent immuno-analyzer, Kaeser 1000), which is expensive for the health services of thirdworld countries. To further overcome these limitations, we present a new way using pulling-force spinning top (PFST) combined with paper-based microfluidic technique to accomplish blood-serum separation and diagnose the SARS-CoV-2 by analyzing the RBD-based $\operatorname{IgA} / \mathrm{IgM} / \mathrm{IgG}$ indicators. This measurement has three great advantages: (1) we proved great specificity and sensitivity of the combined anti-RBD IgA/IgM/ IgG detection, giving a $>99 \%$ accuracy of confirming the SARSCoV-2 infection, and it will be a useful candidate method for clinical RT-PCR diagnosis; (2) people can isolate the serum without any clinical apparatus, and a portable smart phone is easy to record the intensity signal; (3) the manufacturing cost of a single PFST- $\mu$ PADs device is no more than $\$ 5$. These merits provide an economic and reliable solution for both undeveloped and developed countries. We expect this new technology will bring convenience for diagnosis of SARS-CoV-2 and eliminate the dependence on medical apparatus, making the community clinics, family care clinics, and even home diagnosis of SARSCoV-2 feasible.

\section{EXPERIMENTAL SECTION}

Materials. For serum collection, disposable capillaries (YZB, 00022009) were purchased from Yongming Medical Technology, Inc. (Yantai, China); disposable blood needles (Cat\# 01-0628D) were purchased from STERILANCE (Suzhou, China); the spinning top was bought from "children star" toy store at Pinduoduo internet store (PDD.US); and reheating melt rubber gun (model\# RJQHAC50GC113) was purchased from GANCHUN (Shanghai, China). Chitosan (SCR, Cat\# 17779) and glutaraldehyde (SCR, Cat\# 30092436) were purchased from Chemical Reagent Repertory, University of Science and Technology of China. RBD, anti-RBD-IgA, anti-RBD-IgM, and anti-RBD-IgG antibodies were purified in our lab. ${ }^{31}$ HRP-conjugated goat-anti-human IgA (Cat\# BA1066) and HRPconjugated goat-anti-human IgM (Cat\# BA1077) were purchased from Boster Biological Technology Co. (Wuhan, China); goat-anti-human IgG (Lot\# SSA015) was purchased from Sino Biological, Inc. (Beijing, China); HRP-conjugated rabbit anti-goat IgG (Lot\# D110117) was purchased from Sangon Biotech (Shanghai, China), which was also called anti-anti-IgG; and TMB solution (Cat\# P0209) was purchased from Beyotime Biotechnology (Shanghai, China).

Design and Fabrication of $\mu$ PADs. The paper-based device was designed by the illustrator software and fabricated by a wax printing process. The detailed process has been described in the literature. ${ }^{34,35}$ Briefly, the designed patterns were printed on Whatman No. 1 filter paper via a XEROX Phaser $8560 \mathrm{DN}$ wax printer. Then, patterned papers were kept in an oven at $150{ }^{\circ} \mathrm{C}$ for $30 \mathrm{~s}$ to make the wax penetrate the printer completely. To carry out the complex multistep operations, we used plastic comb binding spines (PCBS) valves to control fluid's transportation and perform ELISA assay including antibody immobilization, sample dispensing, and washing.

Paper-Based Chip ELISA Test. Human serums were obtained from three groups of people: SARS-CoV-2-infected group at an early stage (4-10 days), late stage (14-27 days), and healthy control group. Human serum antibodies (anti-RBD-IgA, anti-RBD-IgM, and antiRBD-IgG) were detected. First, $5 \mu \mathrm{L}$ of $0.25 \mathrm{mg} / \mathrm{mL}$ of chitosan was added to every hydrophilic immuno-zone (circle shape) and dried at room temperature. Then, $5 \mu \mathrm{L}$ of $2.5 \%$ of glutaraldehyde dissolved in phosphate-buffered saline (PBS) $(\mathrm{pH}=7.4)$ was added to each immuno-zone. After $2 \mathrm{~h}$ of reaction, immuno-zones were washed with $10 \mu \mathrm{L}$ of $\mathrm{ddH}_{2} \mathrm{O}$ three times. Next, $5 \mu \mathrm{L}$ of RBD (diluted to $2 \mu \mathrm{g} / \mathrm{mL}$ ) was added to immuno-zones at room temperature. After $30 \mathrm{~min}$, immuno-zones were washed with $10 \mu \mathrm{L}$ of PBS three times through PCBS valves. Then, $5 \mu \mathrm{L}$ of $0.5 \%$ bovine serum albumin (BSA) dissolved in PBS ( $\mathrm{pH}=7.4$ ) was added to immuno-zones at room temperature to block the nonspecific binding sites. Immuno-zones were washed three times with PBS 20 min later. After that, $5 \mu \mathrm{L}$ of anti-RBD antibody standards and human serums were added to immuno-zones at room temperature. Anti-RBD antibodies ( $\mathrm{IgA} / \mathrm{IgM} / \mathrm{IgG})$ were doublediluted from 100 to $0 \mathrm{ng} / \mathrm{mL}$ as standards (100, 50, 25, 12.5, 6.25, $0 \mathrm{ng} /$ $\mathrm{mL}$ ). Human serums were diluted 1:100 (healthy people and infected patients) with PBS before adding into the immuno-zones. After $30 \mathrm{~min}$, immuno-zones were washed three times with PBS. Next, $5 \mu \mathrm{L}$ of second antibodies specific binding to anti-RBD IgA $(500 \mathrm{ng} / \mathrm{mL})$, anti-RBD $\operatorname{IgM}(500 \mathrm{ng} / \mathrm{mL})$, or anti-RBD IgG $(500 \mathrm{ng} / \mathrm{mL})$ was added to immuno-zones at room temperature in the dark. After $30 \mathrm{~min}$, the immuno-zones were washed three times with PBS. As second antibodies of IgA and IgM were labeled with HRP, while IgG second antibody did not, $5 \mu \mathrm{L}$ of HRP-labeled IgG third antibody (500 ng/ $\mathrm{mL}$ ) was added to immuno-zones at $4{ }^{\circ} \mathrm{C}$ in the dark and washed after $30 \mathrm{~min}$. After the second and third antibodies were washed, $20 \mu \mathrm{L}$ of TMB solution $\left(3,3^{\prime}, 5,5^{\prime}\right.$-tetramethylbenzidine, substrate of HRP, double-diluted with PBS) was added to each sample and reacted with HRP at room temperature in the dark. After 2 min of chromogenic reaction, the paper-based chips were photographed by a smart phone. 
Any type of smart phone with a good camera is usable. Finally, the pictures were analyzed by ImageJ software, the gray values of immunozones were obtained, and the concentrations of RBD antibodies of the immuno-zones were calculated.

Production of RBD and RBD-Based $\lg A / \lg M / \lg$ S Standards. $\mathrm{RBD}$ and anti-RBD antibodies (IgA/IgM/IgG) were purified as described previously. ${ }^{36}$ Briefly, to make the recombinant SARS-CoV-2 $R B D$, a leader sequence, a sequence encoding spike protein $R B D$, and a human IgG1-Fc were fused together. This sequence was cloned into pTT5 vector and then transiently transfected into HEK293F cells through polyethylenimine. After 3 days, RBD was purified from cell supernatant using protein A column (GE Healthcare). For purification of RBD-based IgA/IgM/IgG, the antigen-immobilized affinity columns were used. The purified RBD was coupled to agarose resin (CNBractivated Sepahrose 4B) according to the manufacturer's protocols (GE Healthcare), packed into an empty column. Next, the antibody standards were purified from patients' serums. Briefly, ammonium sulfate powder was added to the serums and dissolved. After centrifugation, resuspension, and filtration, the patient's serum samples were loaded onto an RBD-Fc affinity column and eluted by linear gradient of elution buffer. The RBD-specific IgG, IgM, and IgA were then purified using a protein $\mathrm{G}$ column and further verified by sodium dodecyl sulfate-polyacrylamide gel electrophoresis (SDS-PAGE) and mass spectrum.

Statistics. Statistics were calculated using Prism5 (GraphPad Software). Group comparisons were analyzed by a two-sided Student's $t$-test. A $p<0.05$ was considered significant. Numbers of asterisks mean significant difference $(* p<0.05 ; * * p<0.01 ; * * * p<0.001 ; * * * p<$ $0.0001)$.

\section{RESULTS AND DISCUSSION}

New Platform for SARS-CoV-2 Diagnosis and $\mu$ PADs Fabrication and Assembly. To diagnose SARS-CoV-2 infection in an efficient and economical way, we designed a new platform as shown in Figure 1. This platform contains a pulling-force spinning top and a $\mu \mathrm{PAD}$ (Figure S1). Because ELISA is a very popular and reliable assay for SARS-CoV-2 detection, our platform used a combination of virus RBDspecific IgA/IgM/IgG antibodies to increase the specificity and sensitivity.

We constructed a paper-based device based on PCBS valves with six parallel channels to perform the ELISA reaction for the detection of SARS-CoV-2. As shown in Figure 1B-E, the chip contains two layers and discontinuous channels. The top layer comprises two long-arm papers, and each component contains six channels. Like flipping calendar, through moving long-arm component, the function of connection (ON state) or disconnection (OFF state) could be achieved using this PCBS. Briefly, when the valves are at an ON state, the longarm paper can connect the middle circles (second layers), which are the immuno-zones, and the liquid could flow from $\mathrm{H}, \mathrm{I}, \mathrm{J}, \mathrm{K}$, $\mathrm{L}, \mathrm{M}$ reservoirs to the $\mathrm{H}^{\prime}, \mathrm{I}^{\prime}, \mathrm{J}^{\prime}, \mathrm{K}^{\prime}, \mathrm{L}^{\prime}, \mathrm{M}^{\prime}$ reservoirs, respectively. In this situation, the residue in the immuno-zones could be washed easily by capillary force (Video S1). Also, at the OFF state, the immuno-zones disconnect from the long-arm paper (Figure 1B-E). Because ELISA reactions generally have multiple processes, such as incubating different immunoreagents, nonspecific-site blocking, multiple washing, etc., the PCBS valves are very convenient for fast diagnosis of SARS$\mathrm{CoV}-2$ at the place without medical equipment.

Isolating the Serum from Blood by the PFST Method. As the spinning top could rotate fast, we considered whether it could be used to separate the human whole blood. The detailed pictures of the spinning top are shown in Figure S2. Whole blood samples from healthy people were exposed from fingers by disposable blood needles and siphoned into capillaries. The
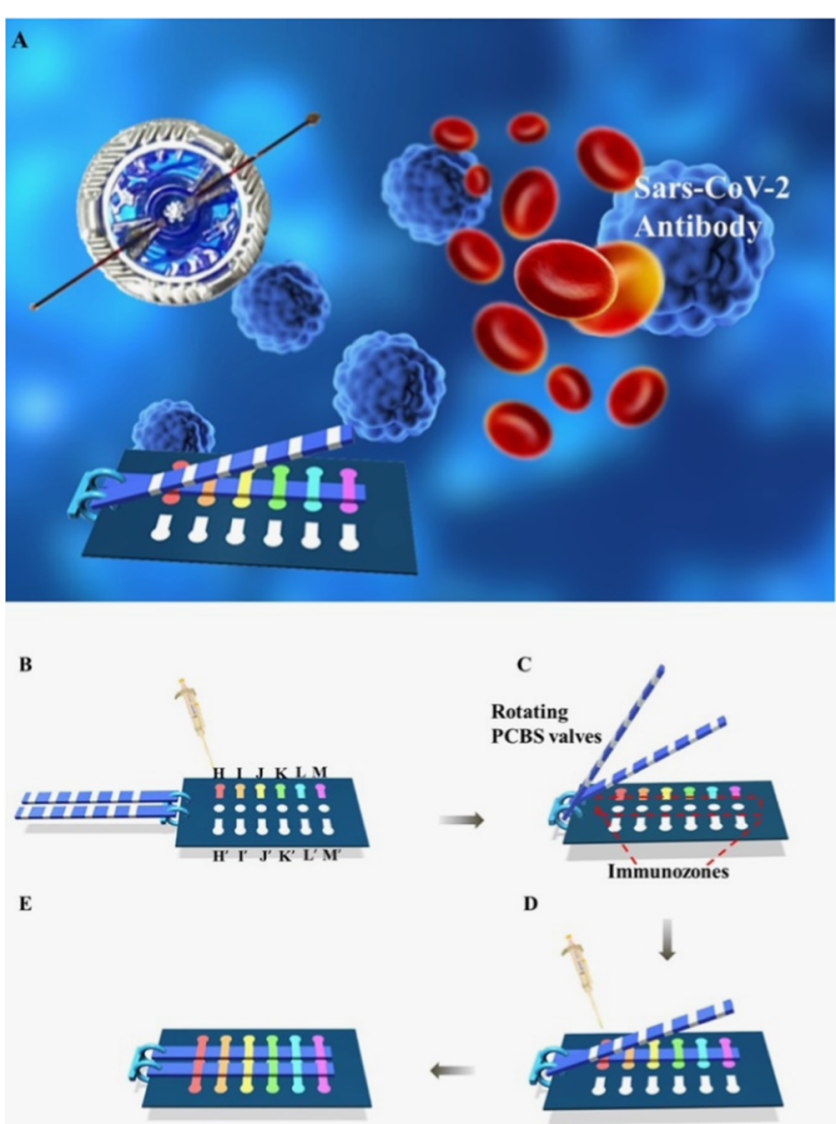

Figure 1. Schematic of the PFST- $\mu$ PADs serological assay. (A) Diagram of PFST- $\mu$ PADs for analyzing SARS-CoV-2. (B) Paper-based valve device has two long-arm channels, ( $\mathrm{H}, \mathrm{I}, \mathrm{J}, \mathrm{K}, \mathrm{L}, \mathrm{M}$ and $\mathrm{H}^{\prime}, \mathrm{I}^{\prime}, \mathrm{J}^{\prime}$, $\mathrm{K}^{\prime}, \mathrm{L}^{\prime}, \mathrm{M}^{\prime}$ ) channels, and six circle reaction zones. ("OFF” state). (C) Rotate long-arm PCBS valves of the paper-based device. (D) H, I, J, K, $\mathrm{L}, \mathrm{M}$ reservoirs connected to the six circle reaction zones. (E) Paperbased valve device closed the long-arm channels ("ON" state), and dyes flowed in the channels.

blood capillary tube has an outer diameter of $1.4 \mathrm{~mm}$, an inner diameter of $0.6 \mathrm{~mm}$, as well as $50 \mathrm{~mm}$ length. Blood was loaded into every capillary, enough for all subsequent analyses on the $\mu$ PADs. One end of capillaries was sealed up by melting glue. Then, capillaries were immediately fixed on the surface of spinning top by melting glue, and the sealed end was against the center of spinning top while the unsealed end was close to the center. About $20 \mu \mathrm{L}$ of whole blood in each capillary tube was separated by PFST. As expected, the volume of serum obtained increased with the accumulated centrifugation time and the amount of serum reached the plateau after 4-5 min (Video S2). The serum was taken out by a Hamilton syringe on the upper layer of capillaries. The average amount of serum collected from each centrifuge tube was about $8 \mu \mathrm{L}$, and this quantity is enough for the following ELISA assays (Figure 2A). It is worth noting that we also addressed the impact of different persons on the isolation of serum in this system. Four different persons used the PFST method to collect the serum from human blood; it took around $4-5 \mathrm{~min}$ for the length of the serum to reach the plateau, indicating that the PFST method is suitable for adults (Figure 2B). Accordingly, for the following experiments, we selected a centrifugation time of $5 \mathrm{~min}$.

We only calculate the maximum speed of the spinning top when it is pulled for the first time and pulled once. If it is pulled 

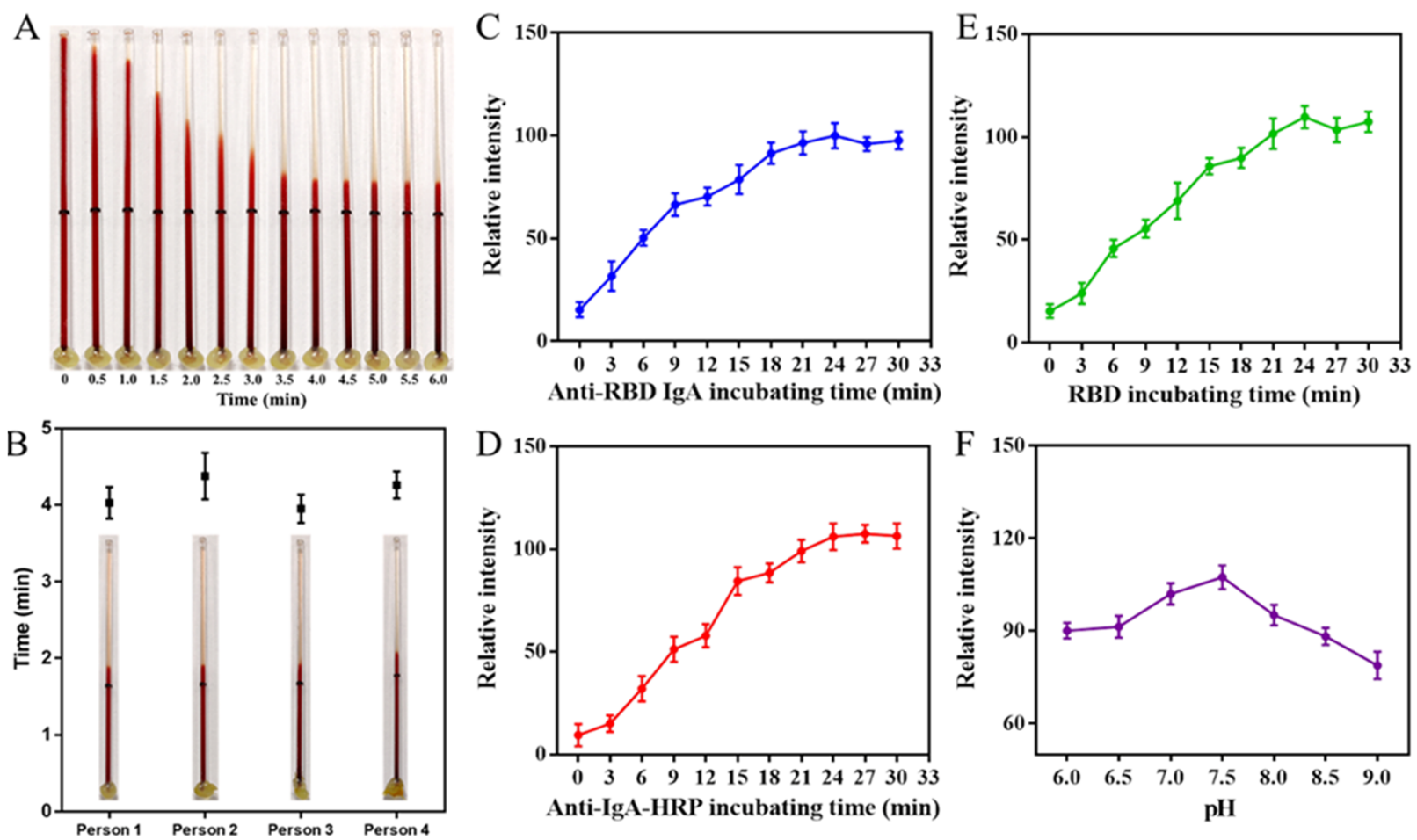

Figure 2. Isolating the serum from whole blood by the PFST method and optimization of experimental parameters controlling the ELISA signals in the PFST- $\mu$ PADs. (A) Kinetics of red blood cells (RBC) and plasma separation. (B) Time for four different persons to isolate the sera from whole blood samples by PFST. Incubation time of anti-RBD-IgA (C), HRP-conjugated Ab (D), and RBD (E), for variation of signal intensity. (F) Variation of signal with different $\mathrm{pH}$ conditions (from 6.0 to 9.0$)(n=3)$.

continuously, the spinning top will reach a higher speed. As shown in Figure S3, when the rope is pulled, the gear transmission is triggered, and the spinning top has begun to rotate. Therefore, the energy of the spinning top comes from pulling the rope. If the friction is ignored, the kinetic energy theory can be obtained

$$
W_{\text {input }}=E_{\mathrm{S}}+E_{\mathrm{A}}+E_{\mathrm{B}}+E_{\mathrm{C}}+E_{\mathrm{D}}
$$

where $W_{\text {input }}$ is the total pulling energy, $E_{\mathrm{S}}$ is the kinetic energy of rope, and A, B, C, and D represent the rope, gear A, gear B, gear $\mathrm{C}$, and the spinning top, respectively (Figure $\mathrm{S} 3$ ). Also, $E_{\mathrm{S}}$ is the kinetic energy of rope; $E_{\mathrm{A}}, E_{\mathrm{B}}$, and $E_{\mathrm{C}}$ are the kinetic energy of gear $A$, gear $B$, and gear $C$, respectively; and $E_{D}$ is the transmission kinetic energy of the spinning top.

Therefore, we can obtain the following equation

$$
F_{\max } \times L_{\mathrm{s}}=\frac{1}{2}\left(m_{\mathrm{s}} v_{\mathrm{s}}^{2}+I_{\mathrm{A}} \omega_{\mathrm{A}}^{2}+I_{\mathrm{B}} \omega_{\mathrm{B}}^{2}+I_{\mathrm{C}} \omega_{\mathrm{C}}^{2}+I_{\mathrm{D}} \omega_{\mathrm{D}}^{2}\right)
$$

where $F_{\max }$ is the maximum pulling force of the rope under normal conditions, $L_{\mathrm{s}}$ is the rope length, $m_{\mathrm{s}}$ is the mass of the rope, $v_{\mathrm{s}}$ is the instantaneous movement speed of the rope, $I$ is the moment of inertia of the gear, and $\omega$ is the instantaneous angular velocity of the gear.

If we regard the gear to be similar to a cylinder, its moment of inertia can be approximately calculated by the existing formula

$$
I_{x}=\frac{1}{2} m_{n} r_{n}^{2}
$$

According to the gear ratio formula

$$
\frac{n_{\mathrm{b}}}{n_{\mathrm{a}}}=\frac{\omega_{\mathrm{a}}}{\omega_{\mathrm{b}}}
$$

where $n$ is the number of gear teeth. And after simplification, we can get the following formula

$$
\begin{aligned}
F_{\max } \times L_{\mathrm{s}}= & \frac{1}{2}\left(\frac{n_{\mathrm{B}} n_{\mathrm{D}} r_{\mathrm{A}}}{3 n_{\mathrm{A}} n_{\mathrm{C}}}\right)^{2} \omega_{\mathrm{D}}^{2}+\frac{1}{2}\left(\frac{n_{\mathrm{B}} n_{\mathrm{D}} r_{\mathrm{A}}}{n_{\mathrm{A}} n_{\mathrm{C}}}\right)^{2} m_{\mathrm{A}} \omega_{\mathrm{D}}^{2} \\
& +\frac{1}{2}\left(\frac{n_{\mathrm{D}} r_{\mathrm{B}}}{n_{\mathrm{C}}}\right)^{2} m_{\mathrm{B}} \omega_{\mathrm{D}}^{2}+\frac{1}{2}\left(\frac{n_{\mathrm{D}} r_{\mathrm{C}}}{n_{\mathrm{C}}}\right)^{2} m_{\mathrm{C}} \omega_{\mathrm{D}}^{2} \\
& \left.+\frac{1}{2} m_{\mathrm{D}} r_{\mathrm{D}}^{2} \omega_{\mathrm{D}}^{2}\right)
\end{aligned}
$$

Using a push-pull tester, the pulling force was around $10 \mathrm{~N} .{ }^{9}$ Using various data as shown in Table S1, we get the maximum angular velocity of the first pulling

$$
\omega_{\mathrm{D}}=753.81 \mathrm{rad} / \mathrm{s}
$$

The approximate value of the theoretical maximum speed that can be obtained for the first pull is

$$
\mathrm{RPM}=\frac{\omega_{\mathrm{D}}}{2 \pi} \approx 120.03 \mathrm{r} / \mathrm{s}=7202.04 \mathrm{rpm}
$$

In fact, if the theoretical speed of the first pulling can reach the speed that satisfies the need of centrifuging serum, then after several pullings, the speed could meet the need of serum separation.

Optimization of the Detection Conditions. As is well known, ELISA performance on the paper chips is also highly related to the parameters such as antigen/antibody incubation time, and $\mathrm{pH}$ value. Therefore, we investigated the different incubation times of primary antibody, HRP-labeled secondary antibody, and RBDs.

The incubation time for the primary antibody and the HRPlabeled secondary antibody (HRP-anti-IgA $500 \mathrm{ng} / \mathrm{mL}$, and 

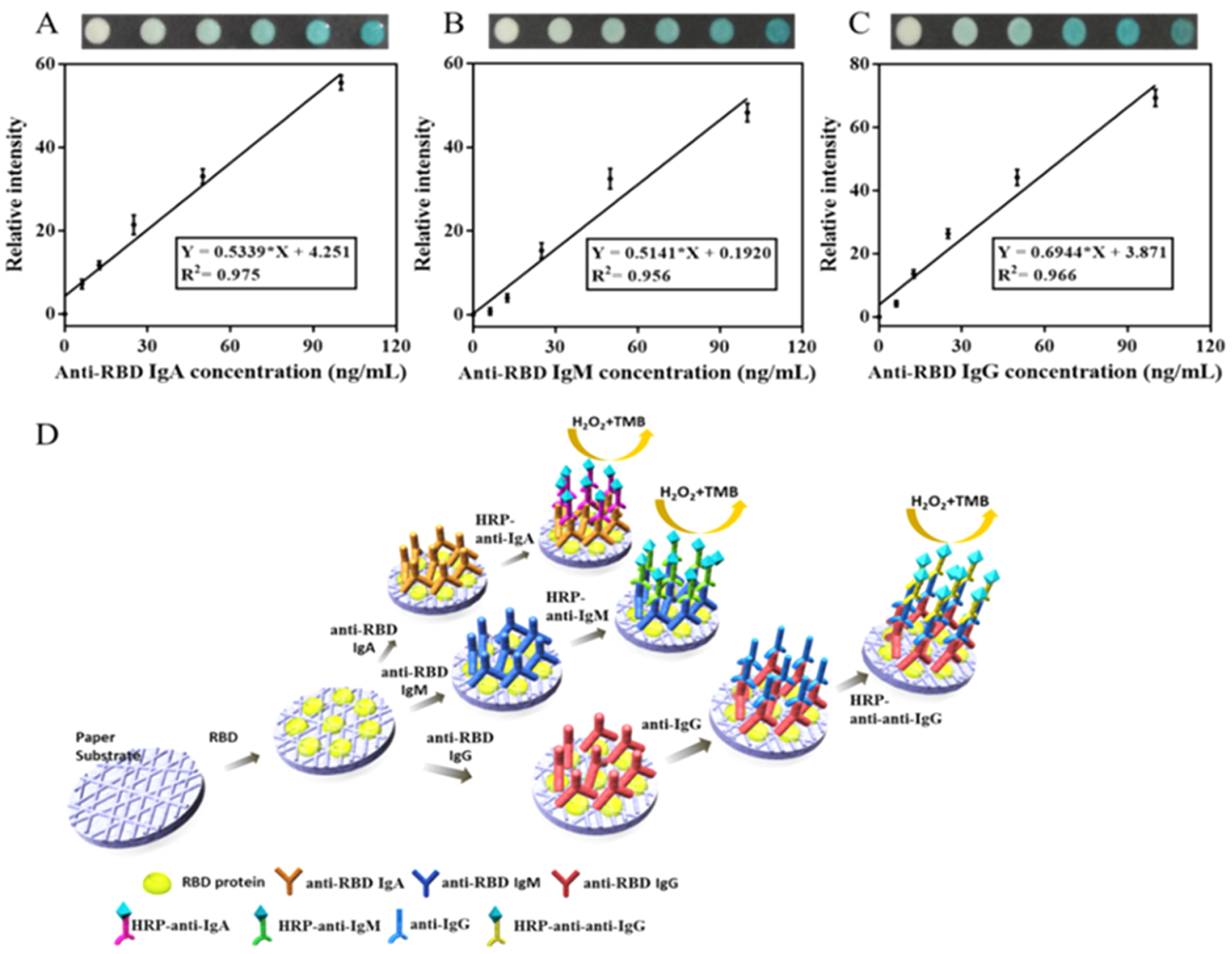

Figure 3. Calibration standard curves for the determination of SARS-CoV-2 RBD-based IgA, IgM, and IgG on $\mu$ PADs. (A-C) The insets show the ELISA results with different IgA, IgM, and IgG concentrations $(100.00,50.00,25.00,12.50,6.25,0 \mathrm{ng} / \mathrm{mL})$ Simple linear regression analysis for IgA, $\operatorname{IgM}$, and IgG $(n=3)$. (D) Schematic diagram of the anti-RBD IgA/IgM/IgG immunoassay procedure on the PFST- $\mu$ PADs. As second antibodies bound to anti-RBD IgA and anti-RBD IgM were labeled with HRP, while those bound to anti-RBD IgG did not, HRP-labeled IgG third antibody (called HRP-anti-anti-IgG) was added.

anti-RBD IgA $100 \mathrm{ng} / \mathrm{mL}$ ) was optimized. As displayed in Figure 2C,D, we observed that the intensity of signal production of ELISA increased with longer incubation time, the intensity of anti-RBD IgA (the prime antibody of IgA) stabilized after an incubation time of $18 \mathrm{~min}$, and the intensity of the HRP-labeled antibody for IgA stabilized after $21 \mathrm{~min}$. To determine the optimal incubation time for the RBD antigen, we studied various incubation times ranging from 0 to $33 \mathrm{~min}$. As shown in Figure $2 \mathrm{E}$, the signals reached a plateau after an incubation time of 20 min. Therefore, we selected $20 \mathrm{~min}$ as a preferred condition.

Due to the great influence of $\mathrm{pH}$ on the quality and intensity of signal production in ELISA assay, we next examined different conditions of wash buffer with $\mathrm{pH}$ value from 6.0 to 9.0. As shown in Figure 2F, the level of anti-RBD IgA peaked at $\mathrm{pH} 7.4$, while under other $\mathrm{pH}$ conditions, the detected intensity signals were all lower than that. Based on this result, we chose $\mathrm{pH} 7.4$ as the standard elution condition for all of the assays in this study. Similarly, under the best $\mathrm{pH}$ condition, incubation times for the primary and secondary antibodies of anti-RBD IgM and antiRBD IgG were as same as that of anti-RBD IgA.

Analytical Performance of PFST- $\mu$ PADs. To characterize the specificity and sensitivity of our $\mu$ PADs, we performed our ELISA immunoassay using the RBD-based $\operatorname{IgA} / \operatorname{IgM} / \operatorname{IgG}$ combination detection system. Briefly, we used our purified recombinant RBD to capture SARS-CoV-2-specific IgA, IgM, and IgG antibodies in patients' serum. We collected these patient-derived IgA/IgM/IgG and prepared our standard for
IgA, IgM, and IgG, which specifically recognize SARS-CoV-2. As our previous report, ${ }^{31}$ we showed 98.2 and $100 \%$ specificity and sensitivity, respectively, in our fluorescence detection system, which could detect the luminescent intensity by an automatic immuno-analyzer. Due to the high cost of this apparatus, we planned to design a new technology with a handy device, with no cost of the luminance machine. To simplify this method, we used the HRP-catalyzed TMB- $\mathrm{H}_{2} \mathrm{O}_{2}$ solution to detect the immune reaction signal, and the signal could be directly photographed by a smart phone. Then, the color could be exchanged to intensity by an open-source tool (ImageJ software). The analytical performance was assessed under optimal conditions through the application of RBD-based IgA/IgM/IgG. Standard solutions of anti-RBD IgA/IgM/IgG (0, 6.25, 12.50, 25.00, 50.00, 100.00 $\mathrm{ng} / \mathrm{mL}$ ) were directly introduced to immune-zones on the paper-based chips. As shown in Figure $3 A-D$, the signal (blue color) increased linearly with IgA, IgM, or IgG concentration between 0 and $100.00 \mathrm{ng} / \mathrm{mL}$. A simple linear regression analysis yielded fits of $y=0.5339 x+4.251\left(R^{2}=0.975\right)$ for anti-RBD $\operatorname{IgA}, y=0.5141 x+0.192\left(R^{2}=0.956\right)$ for anti-RBD IgM, and $y=$ $0.6944 x+3.871\left(R^{2}=0.966\right)$ for anti-RBD IgG, where $y$ is the relative intensity and $x$ represents the protein concentration.

To investigate the reliability of our $\mu \mathrm{PADs}$, we performed a conventional ELISA assay to compare the expression of RBDbased IgA/IgM/IgG, the chemiluminescent intensities of which were detected by a spectrophotometer with the absorbing wavelength at $450 / 595 \mathrm{~nm}$. As shown in Figure S4A-C, a very 
A

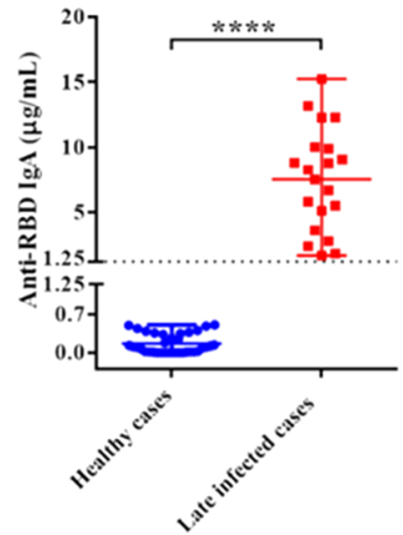

$\mathrm{B}$

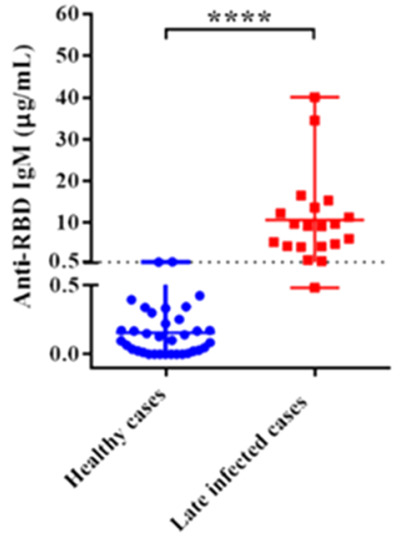

$\mathrm{C}$

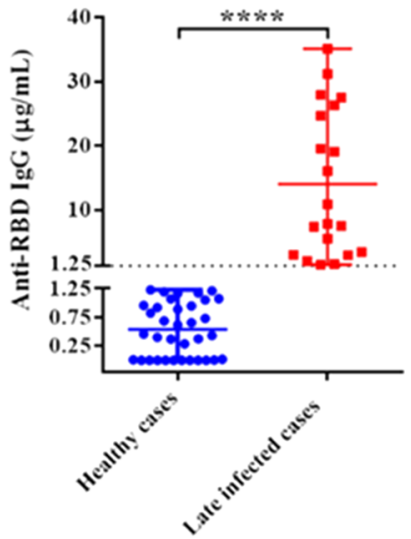

Figure 4. Serum antibody levels in SARS-CoV-2 late-infected patients and healthy people; 20 serums from infected patients and 35 serums from healthy people were collected. Patient samples were collected from 14-27 days post-symptom onset. Antibody levels in serum samples were detected on $\mu$ PAD. For anti-RBD IgA (A), anti-RBD IgM (B), and anti-RBD IgG (C), dash lines were considered as a boundary for distinguishing the infected and healthy people. Data are representative of three independent experiments: $* p<0.05, * * p<0.01, * * * p<0.001, * * * * p<0.0001$.
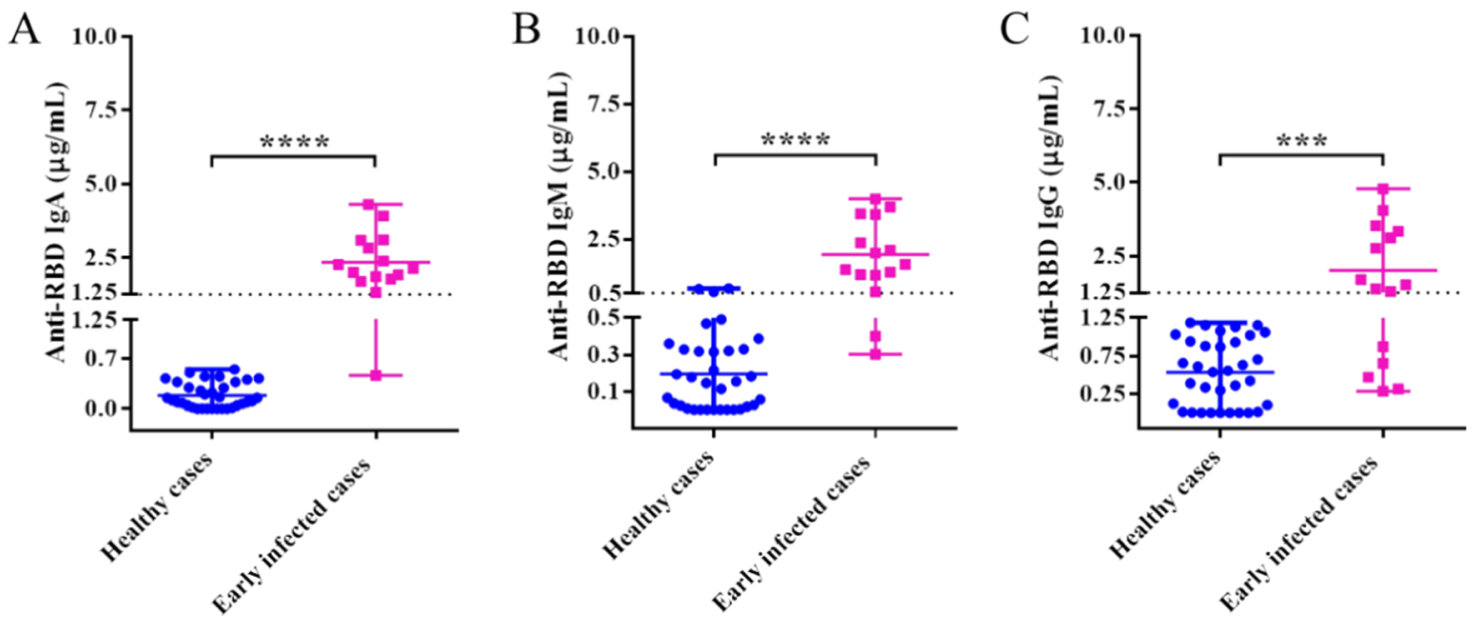

Figure 5. Serum antibody levels in early SARS-CoV-2-infected patients and healthy people; 15 serums from infected patients and 34 serums from healthy people were collected. Patient samples were collected from 4-10 days post-symptom onset. Antibody levels in serum samples were detected on $\mu \mathrm{PAD}$ for anti-RBD IgA (A), anti-RBD IgM (B), and anti-RBD IgG (C); dashed lines were considered as a boundary for distinguishing the infected and uninfected people. Data are representative of three independent experiments: ${ }^{*} p<0.05$, $* * p<0.01, * * * p<0.001, * * * p<0.0001$.

similar pattern of the trend line (slope and $R^{2}$ ) of anti-RBD IgA, $\mathrm{IgM}$, and IgG was observed from $\mu$ PADs results. Based on these findings, we confirmed that the accuracy of our $\mu$ PADs with collecting the digital intensities by smart phone was as good as the conventional ELISA assay detected by a BioTek SYNERGY $\mathrm{H} 1$ microplate reader.

Detecting the Late Stage of SARS-CoV-2-Infected Patient's Serums. To further evaluate the diagnostic accuracy of our PFST- $\mu$ PADs detection method, we measured RBDbased $\operatorname{IgA} / \mathrm{IgM} / \mathrm{IgG}$ production from COVID-19-infected patients compared to healthy people. We collected 55 serum samples from the First Affiliated Hospital of University of Science and Technology of China, including 35 serum samples from healthy people and 20 serum samples from qPCRconfirmed SARS-CoV-2 patients with more than 14 days postsymptom onset (DPSO), considered as late stage of the disease $^{37}$ (Table S2). According to the requirement of the Medical Ethical Committee of the First Affiliated Hospital of University of Science and Technology of China (approval number: $2020-\mathrm{XG}(\mathrm{H})-014)$, to avoid the potential possibility of SARS-CoV-2 spread, all COVID-19-infected patients blood samples were centrifuged at $1500 \mathrm{rpm}$ in test tubes at room temperature for $15 \mathrm{~min}$.

Then, denaturant solution ( $1 \%$ TNBP $+1 \%$ Triton X-100) was added to each tube at $30{ }^{\circ} \mathrm{C}$ for $4 \mathrm{~h}$ to completely denature any potential viruses. And we obtained these deactivated serums of COVID-19 patients. On the other hand, we collected whole blood samples from 35 healthy people and isolated their serums using our PFST method in our lab.

To find the fitful conditions for the good range of serum samples from healthy people and infected patients, we tested the different dilution ratios of these serum samples and found that the intensity of fluorescence showed no decrease up to $1: 20$ dilution, and the signals of $1: 100$ dilution displayed 1/3 peak value (Figure S5A,B). We then choose 1:100 dilution in all of the experiments. All samples were then measured by our paperbased anti-RBD IgA, IgG, and IgM detection method. The results are shown in Figure $4 \mathrm{~A}-\mathrm{C}$, with detailed numbers in Table S3 as well. These three antibodies bind to SARS-CoV-2 $\mathrm{RBD}$ viral antigen with high specificity, which is consistent with our previous report. ${ }^{31}$ Based on the immunological principle, only anti-RBD IgA/IgM/IgG immunoglobin can recognize viral $\mathrm{RBD}$, whereas other nonspecific IgA/IgM/IgG immunoglobin 
Table 1. Comparison of the Sensitivity and Specificity of RBD-Specific IgA, IgM, and IgG as well as Three Different Combinations for SARS-CoV-2 Diagnosis

\begin{tabular}{llllccc} 
& anti-RBD IgA & anti-RBD IgM & anti-RBD IgG & anti-RBD IgA/IgG & anti-RBD IgM/IgG & anti-RBD IgA/IgM/IgG \\
\hline sensitivity (late infected) & $100 \%(20 / 20)$ & $95 \%(19 / 20)$ & $100 \%(20 / 20)$ & $100 \%$ & $100 \%$ \\
specificity (late infected) & $100 \%(35 / 35)$ & $94.3 \%(33 / 35)$ & $100 \%(35 / 35)$ & $100 \%$ & $94.3 \%$ \\
sensitivity (early infected) & $93.3 \%(14 / 15)$ & $86.7 \%(13 / 15)$ & $66.7 \%(10 / 15)$ & $97.80 \%$ & $94.3 \%$ \\
specificity (early infected) & $100 \%(34 / 34)$ & $91.2 \%(31 / 34)$ & $100 \%(34 / 34)$ & $100 \%$ & $95.6 \%$ \\
sensitivity (total) & $97.1 \%(34 / 35)$ & $91.4 \%(32 / 35)$ & $85.7 \%(30 / 35)$ & $99.60 \%$ & $98.7 \%$ \\
specificity (total) & $100 \%(69 / 69)$ & $92.8 \%(64 / 69)$ & $100 \%(69 / 69)$ & $100 \%$ & $91.2 \%$ & $99.9 \%$ \\
\end{tabular}

in the serum cannot. As a result, we could define a threshold line to clearly distinguish healthy people from SARS-CoV-2-infected patients. The threshold lines were the same as our previous finding through a chemical luminescent immuno-analyzer. ${ }^{31}$ After calculations, the threshold lines for the RBD-based IgA/ $\mathrm{IgM} / \mathrm{IgG}$ were $1.25,0.5$, and $1.25 \mu \mathrm{g} / \mathrm{mL}$, respectively, with the corresponding sensitivities of $100 \%(20 / 20), 95 \%(19 / 20)$, and $100 \%(20 / 20)$. For the analysis of clinical samples, the concentration equal to or greater than a cutoff threshold was considered positive. Moreover, the specificities of RBD-based $\operatorname{IgA}$ IgM, and IgG for distinguishing late-infected patients with healthy people were $100 \%(35 / 35), 94.3 \%(33 / 35)$, and $100 \%$ $(35 / 35)$, respectively. These results indicate that both $\operatorname{IgA}$ and IgG display great sensitivity and specificity for diagnosing lateinfected people.

Detecting the Early Stage of SARS-CoV-2-Infected Patient's Serums. As we observed the presence of a high level of RBD-specific IgA in COVID-19 patients' serums. It is well known that during the virus infection, such as EV-71 ${ }^{38}$ and influenza, ${ }^{39}$ a high level of pathogen-specific IgA has been produced. IgA is not only present in the serum of SARS ${ }^{40}$ and COVID- 19 patients, ${ }^{30}$ but also produced by the immune system faster than IgG at the early stage of the disease, suggesting the importance of IgA in immune responses against viral infection. $^{32,33}$

Next, we explored the sensitivity and specificity for these different antibodies at earlier time points to examine whether our IgA/IgM/IgG combined method is good enough for the diagnosis of COVID-19 patients at the early stage of disease development. We collected 49 serum samples from the First Affiliated Hospital of University of Science and Technology of China, including 34 serum samples from healthy people and 15 serum samples from qPCR-confirmed SARS-CoV-2 patients within 4-10 days post-symptom onset, considered as the early stage of the disease ${ }^{36}$ (Table S4). As shown in Figure 5, with detailed numbers in Table S5, the corresponding sensitivities of $\operatorname{IgA}$ IgM, and $\operatorname{IgG}$ were $93.3 \%$ (14/15), 86.7\% (13/15), and $66.7 \%(10 / 15)$. The sensitivity of the $\operatorname{IgA} / \operatorname{IgM} / \operatorname{IgG}$ combination is much higher than the traditional $\operatorname{IgM} / \operatorname{IgG}$ combination (99.7\% vs 95.6\%). On the other hand, both IgA and IgG showed $100 \%(34 / 34)$ specificity for detecting the SARS-CoV-2, while IgM only shows $91.2 \%(31 / 34)$ (Figure 5). Together, IgA test has less false positives, while IgG is not a good marker for people at the early stage of the disease. For IgM, it displays $86.7 \%$ sensitivity and $91.2 \%$ specificity, both lower than those of $\operatorname{IgA}$.

To investigate the reliability of our $\mu \mathrm{PADs}$, we performed a conventional ELISA assay to detect the levels of RBD-based $\operatorname{IgA} / \operatorname{IgM} / \operatorname{IgG}$ by a microplate reader with the absorbing wavelength at $450 / 595 \mathrm{~nm}$. As shown in Figure S6A-C and Table S6, the sensitivities of RBD-based IgA, IgM, and IgG for the late-infected cases were $100 \%(20 / 20), 95 \%(19 / 20)$, and $100 \%(20 / 20)$, respectively, displaying an identical result compared to paper-based microfluidic ELISA. Meanwhile, the specificities for the three antibodies were all 100\% (35/35). A very similar pattern of the early-infected group was displayed (Figure S6D-F). Based on these findings, we confirmed that the accuracy of our $\mu \mathrm{PADs}$ with collecting the digital intensity by a smart phone was as good as the conventional ELISA assay.

To draw a full picture of the effect of $\operatorname{IgA} / \operatorname{IgM} / \operatorname{IgG}$ combination, we calculated all patients' samples (both early and late infected) and healthy people's samples (Table 1). In the strictest way, we regarded serum samples as positive when any levels of IgA, IgM, or IgG over the detection threshold. In this rule, IgA and IgG still showed $100 \%$ specificity for healthy people, while IgM only shows $92.8 \%$ specificity. Because of the existence of $\operatorname{IgM}$, the specificity of $\operatorname{IgA} / \operatorname{IgM} / \operatorname{IgG}$ combination detection is equal to that of $\operatorname{IgM}$ detection alone $(92.8 \%$ vs $92.8 \%)$.

Interestingly, if we choose $\mathrm{IgA} / \mathrm{IgG}$ combination, the specificity is $100 \%$, meaning no false-positive case during the diagnosis (Table 1). As traditional IgM/IgG combination diagnosis is widely used in clinical practice, to avoid divergence and doubt, we still acknowledged the IgA/IgM/IgG combination instead of $\mathrm{IgA} / \mathrm{IgG}$ combination in the serological test.

In the immune system, a large amount of various IgG exists in the body to respond to various antigens. Therefore, we performed a 96-well plate ELISA experiment to detect the concentration of total IgG in the blood of patients as well as healthy people and calculated the percentage of the anti-RBD IgG among total IgG (Figure S7 and Table S7). As the result shows, the total IgG of the patients, especially early-infected patients, was very close to the total $\operatorname{IgG}$ of the healthy people (Figure S7A,B). This result can explain why detecting of serum total IgG always has limited accuracy. Furthermore, we found $0.156 \%$ of anti-RBD IgG/total IgG in late-infected COVID-19 patients, $0.023 \%$ of anti-RBD IgG/total IgG in early-infected patients, and no more than $0.007 \%$ of anti-RBD IgG/total IgG in healthy people (Figure S7C,D and Table S7). This experiment proved that our strategy greatly increased the detection sensitivity of SARS-CoV-2, compared to other clinical ELISA assays examining on total IgG. Moreover, the proposed method by pulling-force-assisted spinning top for blood-serum separation and analysis on microfluidic chip achieved good performance and showed good potential in home diagnosis.

In summary, we highly recommend anti-RBD IgA combined with anti-RBD IgG/IgM method to increase the diagnostic accuracy of SARS-CoV-2 on this microfluidic platform. It displays great diagnostic accuracy for SARS-CoV-2 (99.9\% sensitivity and $92.8 \%$ specificity). Therefore, the use of RBDspecific IgA/IgM/IgG combinational serological test as another solution provides an accurate diagnosis of SARS-CoV-2 on this microfluidic platform. 


\section{DISCUSSION}

In this study, we designed a portable PFST- $\mu$ PAD platform. This device not only shows great sensitivity and specificity for SARSCoV-2 diagnosis but also provides a quantitative anti-RBD IgA/ $\mathrm{IgM} / \mathrm{IgG}$ detection method in an instrument-free way. Recently, our IgA/IgM/IgM combination diagnosis method is recommended by the Canadian Society of Clinical Chemists (CSCC) interim consensus guidance. ${ }^{41}$ More importantly, the novel microfluidic device that separates serum from whole blood and provides quantitative $\mathrm{IgA} / \mathrm{IgM} / \mathrm{IgG}$ combined measurements in a rapid, portable, and potential home diagnosis way. This platform has multiple inherent advantages, such as low costs, small reagent consumption, and no external medical machine needed. It is fit for several application scenarios: (1) undeveloped countries' medical centers, which do not have expensive apparatus; (2) diagnose at developed countries' community clinic to avoid the cross-infection in the crowded hospital; and (3) provide a potential home diagnosis possibility for people who have the experience to take their own blood.

It is well known that IgA is greatly induced during the virus infection, such as EV-71 ${ }^{38}$ and influenza. ${ }^{39}$ During the SARSCoV-2 infection, we previously found IgA and IgM appeared at the early illness onset, while IgG usually emerged 14 days after the onset of symptoms. ${ }^{31}$ We proved the combination of the RBD-based $\operatorname{IgA} / \operatorname{IgM} / \operatorname{IgG}$ giving better diagnosis results compared with the traditional IgM/IgG methods. These come from two immunological principles: one is RBD-specific IgA/ IgM/IgG can improve the accuracy due to its specific recognition of viral RBD, and the other is IgA can be detected both in early and late stages of the disease, while IgG cannot be detected. These data are consistent with another finding that the $\mathrm{IgM}$ and IgG combinatorial detection of SARS-CoV-2 displayed low accuracy for the early stage (1-7 days) and higher accuracy $(96.8 \%)$ at the late stage ( $>15$ days). ${ }^{42}$ Although IgM appears as early as IgA, the sensitivity of IgM for early illness is not accurate compared to IgA diagnosis (Figure 5). This may explain why traditional $\operatorname{IgM} / \operatorname{IgG}$ diagnosis is not accepted by the clinical validation of the COVID-19 patients.

Among 20 PCR-confirmed positive patients at the late stage of the disease and 15 early onsets, we achieved $97.1 \%$ sensitivity and $100 \%$ specificity by anti-RBD IgA detection itself. Other studies also showed the good performance of IgA detection. ${ }^{43,44}$ Thus, we identified that $\operatorname{IgA} / \operatorname{IgM} / \operatorname{IgG}$ combination brought 99.7\% sensitivity for patients at early illness onset compared with traditional IgM/IgG diagnosis, only displaying 95.6\% sensitivity (Table 1).

This study also has some limitations, a major limitation is a low number of patients, and especially we only collect 15 earlyonset patients' samples. Second, as is well known, COVID-19 is a virulent infectious disease, serum inactivation is necessary to prevent the potential risk of infection. We had to obey the requirement of the Medical Ethical Committee of the First Affiliated Hospital of University of Science and Technology of China (approval number: 2020-XG(H)-014 and 2020-p-060), otherwise this study would not be approved. Thus, we only separated the serum from healthy people by the PFST method, not for that from infected people. Finally, to get high-quality results at home by people themselves, it requires these persons to have a basic skill of operating ELISA assay. That is why, we considered our study not only designed a potential home diagnosis method but also provided a new solution for the community clinics, which did not have expensive equipment.
Also, the cost of this paper-based microfluidic ELISA assay is cheaper than conventional ELISA because only $5 \mu \mathrm{L}$ of samples or reagents need to be loaded compared with $50 \mu \mathrm{L}$ volume for conventional ELISA.

Since the appearance of SARS-CoV-2, some rapid detection diagnosis methods were developed, ${ }^{35,45}$ including Cas13-based technique, ${ }^{46}$ DNA nanoscaffold-based assay, ${ }^{49}$ lateral flow immunoassay, ${ }^{48}$ chest CT scan combining a series of X-ray images, ${ }^{49}$ and multiplexed nanomaterial-based sensor array, ${ }^{50}$ but all of these methods neither require an expensive analyzer nor provide quantitative results. Our PFST- $\mu$ PAD method provides a solution for people to isolate their samples and detect a bio-marker of SARS-CoV-2 in a very convenient and portable way without any clinical apparatus, which makes home diagnosis achievable and available. Moreover, our detection system shows high specificity and sensitivity to SARS-CoV-2 infection, indicating that the PFST- $\mu$ PAD-based detection of anti-RBD $\operatorname{IgA} / \operatorname{IgM} / \operatorname{IgG}$ might be a fast, cheap, and facile way for SARSCoV-2 diagnosis.

\section{CONCLUSIONS}

In summary, we reported a novel and handy way to isolate the serum from whole blood by the PFST method and detect the $\mathrm{RBD}$-based $\mathrm{IgA} / \mathrm{IgM} / \mathrm{IgG}$ concentrations by $\mu \mathrm{PADs}$. It provides an affordable, rapid, and user-friendly way to the diagnosis of COVID-19 in a microfluidic format. By the combination of the viral RBD-specific antibodies (anti-RBD $\operatorname{IgA} / \operatorname{IgM} / \operatorname{IgG}$ ), our methods displayed great sensitive and quantitative serological results. It is worth noting that our PFST$\mu$ PADs system can also be used for the diagnosis of COVID-19 patients both at the early and late stages of the disease. Moreover, no need for the clinical apparatus and low cost of the portable device give a new solution for both developed and undeveloped countries to monitor the spread of the COVID-19 disease in a potential home diagnosis pattern.

\section{ASSOCIATED CONTENT}

\section{Supporting Information}

The Supporting Information is available free of charge at https://pubs.acs.org/doi/10.1021/acssensors.1c00773.

PFST- $\mu$ PADs detection platform; photographs of the spinning top with its handle; picture of the gears A, B and $\mathrm{C}$, spinning top, and the rope inside the handle; canonical ELISA standard curves for human RBD antibodies concentration on 96-well plate; option of dilution ratio of SARS-CoV-2 patients serums; serum antibody levels in SARS-CoV-2 patients and healthy people in conventional ELISA detection; serum total IgG concentration and the proportion of the anti-RBD IgG among total IgG in SARS-CoV-2 patients and healthy people; information of four gears and the pulling rope of the spinning top; information of the 20 late-stage COVID-19 patients (confirmed for 14-27 days) from the First Affiliated Hospital of University of Science and Technology of China; 20 late-stage COVID-19 patients and 35 healthy people serum samples and their anti-RBD IgA, anti-RBD IgM, and anti-RBD IgG determination results; information of 15 early-stage COVID-19 patients (confirmed for 4-10 days) from the First Affiliated Hospital of University of Science and Technology of China; 15 early-stage COVID-19 patients and 34 healthy people serum samples and their anti-RBD IgA, anti-RBD IgM, 
and anti-RBD IgG determination results; sensitivity and specificity of RBD-specific $\operatorname{IgA}, \operatorname{IgM}$, and IgG, as well as three different combinations for diagnosing in conventional ELISA detection; and the serum total IgG concentration determination results and the proportion of the anti-RBD IgG among total IgG in the early- and late-infected patients and healthy people (PDF)

Entire fabrication process of PCBS valves (Video S1) (MP4)

Serum collection by the PFST method (Video S2) (MP4)

\section{AUTHOR INFORMATION}

\section{Corresponding Authors}

Tengchuan Jin - Department of Medical Oncology, The First Affiliated Hospital of USTC, Division of Life Sciences and Medicine, University of Science and Technology of China, Hefei 230001, China; (1) orcid.org/0000-0002-1395-188X; Email: jint@ustc.edu.cn

Bowei Li - CAS Key Laboratory of Coastal Environmental Processes and Ecological Remediation, Research Center for Coastal Environmental Engineering and Technology, Yantai Institute of Coastal Zone Research, Chinese Academy of Sciences, Yantai 264003, China; (1) orcid.org/0000-00016262-8248; Email: bwli@yic.ac.cn

Bofeng Li - Department of Medical Oncology, The First Affiliated Hospital of USTC, Division of Life Sciences and Medicine, University of Science and Technology of China, Hefei 230001, China; O orcid.org/0000-0002-9110-8804; Email: libf@ustc.edu.cn

\section{Authors}

Fanwu Gong - Department of Medical Oncology, The First Affiliated Hospital of USTC, Division of Life Sciences and Medicine, University of Science and Technology of China, Hefei 230001, China

Hua-xing Wei - Department of Laboratory Medicine, The First Affiliated Hospital of USTC, Division of Life Sciences and Medicine, University of Science and Technology of China, Hefei 230001, China

Ji Qi - CAS Key Laboratory of Coastal Environmental Processes and Ecological Remediation, Research Center for Coastal Environmental Engineering and Technology, Yantai Institute of Coastal Zone Research, Chinese Academy of Sciences, Yantai 264003, China

Huan Ma - Department of Medical Oncology, The First Affiliated Hospital of USTC, Division of Life Sciences and Medicine, University of Science and Technology of China, Hefei 230001, China

Lianxin Liu - Department of Hepatobiliary Surgery, The First Affiliated Hospital of USTC, Division of Life Sciences and Medicine, University of Science and Technology of China, Hefei 230021, China

Jianping Weng - Department of Endocrinology and Metabolism, The First Affiliated Hospital of USTC, Division of Life Sciences and Medicine, University of Science and Technology of China, Hefei 230027, China

Xucai Zheng - Department of Medical Oncology, The First Affiliated Hospital of USTC, Division of Life Sciences and Medicine, University of Science and Technology of China, Hefei 230001, China

Qiangsheng Li - Department of Medical Oncology, The First Affiliated Hospital of USTC, Division of Life Sciences and
Medicine, University of Science and Technology of China, Hefei 230001, China

Dan Zhao - Department of Medical Oncology, The First Affiliated Hospital of USTC, Division of Life Sciences and Medicine, University of Science and Technology of China, Hefei 230001, China

Haopeng Fang - Department of Medical Oncology, The First Affiliated Hospital of USTC, Division of Life Sciences and Medicine, University of Science and Technology of China, Hefei 230001, China

Liu Liu - Department of Medical Oncology, The First Affiliated Hospital of USTC, Division of Life Sciences and Medicine, University of Science and Technology of China, Hefei 230001, China

Hongliang He - Department of Laboratory Medicine, The First Affiliated Hospital of USTC, Division of Life Sciences and Medicine, University of Science and Technology of China, Hefei 230001, China

Cuichen Ma - Department of Medical Oncology, The First Affiliated Hospital of USTC, Division of Life Sciences and Medicine, University of Science and Technology of China, Hefei 230001, China

Jinglong Han - School of Environment and Materials Engineering, Yantai University, Yantai 264005, China

Anyuan Sun - Department of Laboratory Medicine, The First Affiliated Hospital of USTC, Division of Life Sciences and Medicine, University of Science and Technology of China, Hefei 230001, China

Baolong Wang - Department of Laboratory Medicine, The First Affiliated Hospital of USTC, Division of Life Sciences and Medicine, University of Science and Technology of China, Hefei 230001, China

Complete contact information is available at:

https://pubs.acs.org/10.1021/acssensors.1c00773

\section{Author Contributions}

Bowei Li, T.J., and Bofeng Li designed the research. F.G., H.M., J.Q., Q.L., H.F., and D.Z. performed the research. F.G., C.M., J.H., Bowei Li, and J.Q. analyzed data. Bofeng Li and H.-x.W. wrote the paper. X.Z., A.S., B.W., Liu Liu, H.H., J.W., and Lianxin Liu provided blood samples and clinical information of COVID-19 patients and healthy people.

\section{Notes}

The authors declare no competing financial interest.

\section{ACKNOWLEDGMENTS}

This work was supported by the National Natural Science Foundation of China (Grant Nos. 41776110, 31870731, and 81974258), COVID-19 special task grant from Chinese Academy of Science Clinical Research Hospital (Hefei) (YD2070002017 and YD9110002006), Shandong Provincial Natural Science Foundation Key Project (ZR2020KB022), and China Postdoctoral Science Foundation (2020M670084ZX).

\section{REFERENCES}

(1) Jones, D. S. History in a Crisis - Lessons for Covid-19. N. Engl. J. Med. 2020, 382, 1681-1683.

(2) Cui, J.; Li, F.; Shi, Z. L. Origin and evolution of pathogenic coronaviruses. Nat. Rev. Microbiol. 2019, 17, 181-192.

(3) Zhu, N.; Zhang, D.; Wang, W.; Li, X.; Yang, B.; Song, J.; Zhao, X.; Huang, B.; Shi, W.; Lu, R.; Niu, P.; Zhan, F.; Ma, X.; Wang, D.; Xu, W.; Wu, G.; Gao, G. F.; Tan, W.; China Novel Coronavirus Investigating 
and Research Team. A Novel Coronavirus from Patients with Pneumonia in China, 2019. N. Engl. J. Med. 2020, 382, 727-733.

(4) Subbarao, K.; Mahanty, S. Respiratory Virus Infections: Understanding COVID-19. Immunity 2020, 52, 905-909.

(5) Bastos, M. L.; Tavaziva, G.; Abidi, S. K.; Campbell, J. R.; Haraoui, L. P.; Johnston, J. C.; Lan, Z.; Law, S.; MacLean, E.; Trajman, A.; Menzies, D.; Benedetti, A.; Ahmad Khan, F. Diagnostic accuracy of serological tests for covid-19: systematic review and meta-analysis. BMJ 2020, 370, No. m2516.

(6) Winichakoon, P.; Chaiwarith, R.; Liwsrisakun, C.; Salee, P.; Goonna, A.; Limsukon, A.; Kaewpoowat, Q. Negative Nasopharyngeal and Oropharyngeal Swabs Do Not Rule Out COVID-19. Negative Nasopharyngeal and Oropharyngeal Swabs Do Not Rule Out COVID19. J. Clin. Microbiol. 2020, 58, No. e00297-20.

(7) Florindo, H. F.; Kleiner, R.; Vaskovich-Koubi, D.; Acurcio, R. C.; Carreira, B.; Yeini, E.; Tiram, G.; Liubomirski, Y.; Satchi-Fainaro, R. Immune-mediated approaches against COVID-19. Nat. Nanotechnol. 2020, 15, 630-645.

(8) Maxmen, A. The researchers taking a gamble with antibody tests for coronavirus. Nature 2020, DOI: 10.1038/d41586-020-01163-5.

(9) Li, B.; Qi, J.; Fu, L.; Han, J.; Choo, J.; deMello, A. J.; Lin, B.; Chen, L. Integrated hand-powered centrifugation and paper-based diagnosis with blood-in/answer-out capabilities. Biosens. Bioelectron. 2020, 165, No. 112282

(10) Bai, R.; Li, L.; Liu, M.; Yan, S.; Miao, C.; Li, R.; Luo, Y.; Liu, T.; Lin, B.; Ji, Y.; Lu, Y. Paper-Based 3D Scaffold for Multiplexed Single Cell Secretomic Analysis. Anal. Chem. 2018, 90, 5825-5832.

(11) Song, Y. L.; Lin, B. Q.; Tian, T.; Xu, X.; Wang, W.; Ruan, Q. Y.; Guo, J. J.; Zhu, Z.; Yang, C. Y. Recent Progress in Microfluidics-Based Biosensing. Anal. Chem. 2019, 91, 388-404.

(12) Mahato, K.; Srivastava, A.; Chandra, P. Paper based diagnostics for personalized health care: Emerging technologies and commercial aspects. Biosens. Bioelectron. 2017, 96, 246-259.

(13) Huang, X.; Xu, D.; Chen, J.; Liu, J.; Li, Y.; Song, J.; Ma, X.; Guo, J. Smartphone-based analytical biosensors. Analyst 2018, 143, 53395351.

(14) Qi, J.; Li, B.; Wang, X.; Zhang, Z.; Wang, Z.; Han, J.; Chen, L. Three-dimensional paper-based microfluidic chip device for multiplexed fluorescence detection of $\mathrm{Cu}^{2+}$ and $\mathrm{Hg}^{2+}$ ions based on ion imprinting technology. Sens. Actuators, B 2017, 251, 224-233.

(15) Liu, D.; Li, X.; Zhou, J.; Liu, S.; Tian, T.; Song, Y.; Zhu, Z.; Zhou, L.; Ji, T.; Yang, C. A fully integrated distance readout ELISA-Chip for point-of-care testing with sample-in-answer-out capability. Biosens. Bioelectron. 2017, 96, 332-338.

(16) Salentijn, G. I. J.; Grajewski, M.; Verpoorte, E. Reinventing (Bio)chemical Analysis with Paper. Anal. Chem. 2018, 90, 1381513825 .

(17) Ge, L.; Yan, J.; Song, X.; Yan, M.; Ge, S.; Yu, J. Three-dimensional paper-based electrochemiluminescence immunodevice for multiplexed measurement of biomarkers and point-of-care testing. Biomaterials 2012, 33, 1024-1031.

(18) Li, L.; Zhang, Y.; Zhang, L.; Ge, S.; Liu, H.; Ren, N.; Yan, M.; Yu, J. Paper-Based Device for Colorimetric and Photoelectrochemical Quantification of the Flux of $\mathrm{H} 2 \mathrm{O} 2$ Releasing from MCF-7 Cancer Cells. Anal. Chem. 2016, 88, 5369-5377.

(19) Wang, S.; Ge, L.; Song, X.; Yu, J.; Ge, S.; Huang, J.; Zeng, F. Paper-based chemiluminescence ELISA: Lab-on-paper based on chitosan modified paper device and wax-screen-printing. Biosens. Bioelectron. 2012, 31, 212-218.

(20) Bhamla, M. S.; Benson, B.; Chai, C.; Katsikis, G.; Johri, A.; Prakash, M. Hand-powered ultralow-cost paper centrifuge. Nat. Biomed. Eng. 2017, 1, No. 0009.

(21) Michael, I.; Kim, D.; Gulenko, O.; Kumar, S.; Kumar, S.; Clara, J.; Ki, D. Y.; Park, J.; Jeong, H. Y.; Kim, T. S.; Kwon, S.; Cho, Y. K. A fidget spinner for the point-of-care diagnosis of urinary tract infection. Nat. Biomed. Eng. 2020, 4, 591-600.

(22) Liu, C. H.; Chen, C. A.; Chen, S. J.; Tsai, T. T.; Chu, C. C.; Chang, C. C.; Chen, C. F. Blood Plasma Separation Using a FidgetSpinner. Anal. Chem. 2019, 91, 1247-1253.
(23) Kim, D.; Lee, J. Y.; Yang, J. S.; Kim, J. W.; Kim, V. N.; Chang, H. The Architecture of SARS-CoV-2 Transcriptome. Cell 2020, 181, 914921.

(24) Hoffmann, M.; Kleine-Weber, H.; Schroeder, S.; Kruger, N.; Herrler, T.; Erichsen, S.; Schiergens, T. S.; Herrler, G.; Wu, N. H.; Nitsche, A.; Muller, M. A.; Drosten, C.; Pohlmann, S. SARS-CoV-2 Cell Entry Depends on ACE2 and TMPRSS2 and Is Blocked by a Clinically Proven Protease Inhibitor. Cell 2020, 181, 271-280.

(25) Lan, J.; Ge, J.; Yu, J.; Shan, S.; Zhou, H.; Fan, S.; Zhang, Q.; Shi, X.; Wang, Q.; Zhang, L.; Wang, X. Structure of the SARS-CoV-2 spike receptor-binding domain bound to the ACE2 receptor. Nature 2020, 581, 215-220.

(26) Cao, X. COVID-19: immunopathology and its implications for therapy. Nat. Rev. Immunol. 2020, 20, 269-270.

(27) Schroeder, H. W., Jr.; Cavacini, L. Structure and function of immunoglobulins. J. Allergy Clin. Immunol. 2010, 125, S41-S52.

(28) García, L. F. Immune Response, Inflammation, and the Clinical Spectrum of COVID-19. Front. Immunol. 2020, 11, No. 1441.

(29) Boes, M. Role of natural and immune IgM antibodies in immune responses. Mol. Immunol. 2000, 37, 1141-1149.

(30) Dan, L.; Liu, L.; Sun, Y.; Song, J.; Yin, Q.; Zhang, G.; Qi, F.; Hu, Z.; Yang, Z.; Zhou, Z.; Hu, Y.; Zhang, L.; Ji, J.; Zhao, X.; Jin, Y.; McNutt, M. A.; Yin, Y. The phosphatase PAC1 acts as a T cell suppressor and attenuates host antitumor immunity. Nat. Immunol. 2020, 21, 287297.

(31) Ma, H.; Zeng, W.; He, H.; Zhao, D.; Jiang, D.; Zhou, P.; Cheng, L.; Li, Y.; Ma, X.; Jin, T. Serum IgA, IgM, and IgG responses in COVID19. Cell. Mol. Immunol. 2020, 17, 773-775.

(32) Guo, L.; Ren, L.; Yang, S.; Xiao, M.; Chang; Yang, F.; Dela Cruz, C. S.; Wang, Y.; Wu, C.; Xiao, Y.; Zhang, L.; Han, L.; Dang, S.; Xu, Y.; Yang, Q. W.; Xu, S. Y.; Zhu, H. D.; Xu, Y. C.; Jin, Q.; Sharma, L.; Wang, L.; Wang, J. Profiling Early Humoral Response to Diagnose Novel Coronavirus Disease (COVID-19). Clin. Infect. Dis. 2020, 71, 778-785.

(33) Huang, Z.; Chen, H.; Xue, M.; Huang, H.; Zheng, P.; Luo, W.; Liang, X.; Sun, B.; Zhong, N. Characteristics and roles of SARS-CoV-2 specific antibodies in patients with different severities of COVID-19. Clin. Exp. Immunol. 2020, 202, 210-219.

(34) Qi, J.; Li, B.; Zhou, N.; Wang, X.; Deng, D.; Luo, L.; Chen, L. The strategy of antibody-free biomarker analysis by in-situ synthesized molecularly imprinted polymers on movable valve paper-based device. Biosens. Bioelectron. 2019, 142, No. 111533.

(35) Zhou, J.; Li, B.; Qi, A.; Shi, Y.; Qi, J.; Xu, H.; Chen, L. ZnSe quantum dot based ion imprinting technology for fluorescence detecting cadmium and lead ions on a three-dimensional rotary paper-based microfluidic chip. Sens. Actuators, B 2020, 305, No. 127462.

(36) Zeng, W.; Ma, H.; Ding, C.; Yang, Y.; Sun, Y.; Huang, X.; He, W.; Xiang, Y.; Gao, Y.; Jin, T. Characterization of SARS-CoV-2-specific antibodies in COVID-19 patients reveals highly potent neutralizing IgA. Signal Transduction Targeted Ther. 2021, 6, No. 35.

(37) Pisanic, N.; Randad, P. R.; Kruczynski, K.; Manabe, Y. C.; Thomas, D.; Pekosz, A.; Klein, S. L.; Betenbaugh, M. J.; Clarke, W. A.; Laeyendecker, O.; Caturegli, P. P.; Larman, H. B.; Detrick, B.; Fairley, J. K.; Sherman, A. C.; Rouphael, N.; Edupuganti, S.; Granger, D. A.; Granger, S. W.; Collins, M.; Heaney, C. D. COVID-19 Serology at Population Scale: SARS-CoV-2-Specific Antibody Responses in Saliva. J. Clin. Microbiol. 2021, 59, No. e02204-20.

(38) Lin, Y. L.; Cheng, P. Y.; Chin, C. L.; Huang, L. M.; Lin, S. Y.; Chiang, B. L. Fibroblast-stimulating lipopeptide-1 as a potential mucosal adjuvant enhances mucosal and systemic immune responses to enterovirus 71 vaccine. Vaccine 2018, 36, 4331-4338.

(39) Grund, S.; Michel, S.; Barthuber, C.; Adams, O. Serum and mucosal antibodies fail as prognostic markers during critical influenza $\mathrm{A}$ infection. J. Clin. Virol. 2016, 74, 32-36.

(40) Wu, L. P.; Wang, N. C.; Chang, Y. H.; Tian, X. Y.; Na, D. Y.; Zhang, L. Y.; Zheng, L.; Lan, T.; Wang, L. F.; Liang, G. D. Duration of antibody responses after severe acute respiratory syndrome. Emerg. Infect. Dis. 2007, 13, 1562-1564. 
(41) Bailey, D.; Konforte, D.; Barakauskas, V. E.; Yip, P. M.; Kulasingam, V.; Abou El Hassan, M.; Beach, L. A.; Blasutig, I. M.; Catomeris, P.; Dooley, K. C.; Gong, Y. P.; Kavsak, P.; Randell, E. W.; Robinson, J. L.; Shaw, J.; Taher, J.; White-Al Habeeb, N. Canadian society of clinical chemists (CSCC) interim consensus guidance for testing and reporting of SARS-CoV-2 serology. Clin. Biochem. 2020, 86, $1-7$.

(42) Pan, Y.; Li, X.; Yang, G.; Fan, J.; Tang, Y.; Zhao, J.; Long, X.; Guo, S.; Zhao, Z.; Liu, Y.; Hu, H.; Xue, H.; Li, Y. Serological immunochromatographic approach in diagnosis with SARS-CoV-2 infected COVID-19 patients. J. Infect. 2020, 81, e28-e32.

(43) GeurtsvanKessel, C. H.; Okba, N. M. A.; Igloi, Z.; Bogers, S.; Embregts, C. W. E.; Laksono, B. M.; Leijten, L.; Rokx, C.; Rijnders, B.; Rahamat-Langendoen, J.; van den Akker, J. P. C.; van Kampen, J. J. A.; van der Eijk, A. A.; van Binnendijk, R. S.; Haagmans, B.; Koopmans, M. An evaluation of COVID-19 serological assays informs future diagnostics and exposure assessment. Nat. Commun. 2020, 11, No. 3436.

(44) Tré-Hardy, M.; Wilmet, A.; Beukinga, I.; Favresse, J.; Dogne, J. M.; Douxfils, J.; Blairon, L. Analytical and clinical validation of an ELISA for specific SARS-CoV-2 IgG, IgA, and IgM antibodies. J. Med. Virol. 2021, 93, 803-811.

(45) Cui, F.; Zhou, H. S. Diagnostic methods and potential portable biosensors for coronavirus disease 2019. Biosens. Bioelectron. 2020, 165, No. 112349.

(46) Patchsung, M.; Jantarug, K.; Pattama, A.; Aphicho, K.; Suraritdechachai, S.; Meesawat, P.; Sappakhaw, K.; Leelahakorn, N.; Ruenkam, T.; Wongsatit, T.; Athipanyasilp, N.; Eiamthong, B.; Lakkanasirorat, B.; Phoodokmai, T.; Niljianskul, N.; Pakotiprapha, D.; Chanarat, S.; Homchan, A.; Tinikul, R.; Kamutira, P.; Phiwkaow, K.; Soithongcharoen, S.; Kantiwiriyawanitch, C.; Pongsupasa, V.; Trisrivirat, D.; Jaroensuk, J.; Wongnate, T.; Maenpuen, S.; Chaiyen, P.; Kamnerdnakta, S.; Swangsri, J.; Chuthapisith, S.; Sirivatanauksorn, Y.; Chaimayo, C.; Sutthent, R.; Kantakamalakul, W.; Joung, J.; Ladha, A.; Jin, X.; Gootenberg, J. S.; Abudayyeh, O. O.; Zhang, F.; Horthongkham, N.; Uttamapinant, C. Clinical validation of a Cas13based assay for the detection of SARS-CoV-2 RNA. Nat. Biomed. Eng. 2020, 4, 1140-1149.

(47) Jiao, J.; Duan, C.; Xue, L.; Liu, Y.; Sun, W.; Xiang, Y. DNA nanoscaffold-based SARS-CoV-2 detection for COVID-19 diagnosis. Biosens. Bioelectron. 2020, 167, No. 112479.

(48) Wang, D.; He, S.; Wang, X.; Yan, Y.; Liu, J.; Wu, S.; Liu, S.; Lei, Y.; Chen, M.; Li, L.; Zhang, J.; Zhang, L.; Hu, X.; Zheng, X.; Bai, J.; Zhang, Y.; Zhang, Y.; Song, M.; Tang, Y. Rapid lateral flow immunoassay for the fluorescence detection of SARS-CoV-2 RNA. Nat. Biomed. Eng. 2020, 4, 1150-1158.

(49) Bai, H. X.; Hsieh, B.; Xiong, Z.; Halsey, K.; Choi, J. W.; Tran, T. M. L.; Pan, I.; Shi, L. B.; Wang, D. C.; Mei, J.; Jiang, X. L.; Zeng, Q. H.; Egglin, T. K.; Hu, P. F.; Agarwal, S.; Xie, F. F.; Li, S.; Healey, T.; Atalay, M. K.; Liao, W. H. Performance of Radiologists in Differentiating COVID-19 from Non-COVID-19 Viral Pneumonia at Chest CT. Radiology 2020, 296, E46-E54.

(50) Shan, B.; Broza, Y. Y.; Li, W.; Wang, Y.; Wu, S.; Liu, Z.; Wang, J.; Gui, S.; Wang, L.; Zhang, Z.; Liu, W.; Zhou, S.; Jin, W.; Zhang, Q.; Hu, D.; Lin, L.; Zhang, Q.; Li, W.; Wang, J.; Liu, H.; Pan, Y.; Haick, H. Multiplexed Nanomaterial-Based Sensor Array for Detection of COVID-19 in Exhaled Breath. ACS Nano 2020, 14, 12125-12132. 